\title{
The luminosity function of high-redshift quasi-stellar objects. A combined analysis of GOODS and SDSS ${ }^{\star}$
}

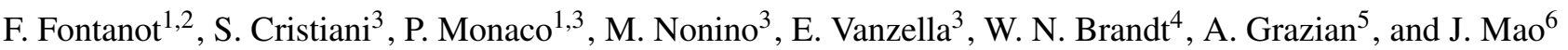 \\ 1 Dipartimento di Astronomia dell'Università, Via Tiepolo 11, 34131 Trieste, Italy \\ e-mail: fontanot@mpia.de \\ 2 Max-Planck-Institut for Astronomy, Koenigstuhl 17, 69117 Heidelberg, Germany \\ e-mail: fontanot@mpia.de \\ 3 INAF-Osservatorio Astronomico, via Tiepolo 11, 34131 Trieste, Italy \\ 4 Department of Astronomy and Astrophysics, Pennsylvania State University, 525 Davey Lab, University Park, PA 16802, USA \\ 5 INAF-Osservatorio Astronomico di Roma, via Frascati 33, 00040 Monteporzio, Italy \\ ${ }^{6}$ SISSA, via Beirut 2-4, 34014 Trieste, Italy
}

Received 20 July 2006 / Accepted 30 August 2006

\section{ABSTRACT}

\begin{abstract}
Aims. In this work the luminosity function of QSOs is measured in the redshift range $3.5<z<5.2$ for the absolute magnitude interval $-21<M_{145}<-28$. Determining the faint-end of the luminosity function at these redshifts provides important constraints on models of the joint evolution of galaxies and AGNs.

Methods. We have defined suitable criteria to select faint QSOs in the GOODS fields, checking their effectiveness and completeness in detail. A spectroscopic follow-up of the resulting QSO candidates was carried out. The confirmed sample of faint QSOs is compared with a brighter one derived from the SDSS. We used a Monte-Carlo technique to estimate the properties of the luminosity function, checking various parameterizations for its shape and evolution.

Results. Models based on pure density evolution show better agreement with observation than do models based on pure luminosity evolution. However, a different break magnitude with respect to $z \sim 2.1$ is required at $3.5<z<5.2$. Models with a steeper faint-end score a higher probability. We do not find any evidence for a bright-end flattening at redshift $z>3.5$.

Conclusions. The estimated space density evolution of QSOs indicates a suppression of the formation and/or feeding of supermassive black holes at these redshifts. The QSO contribution to the UV background is insufficient for ionizing the IGM at $3.5<z<5.2$.
\end{abstract}

Key words. quasars: general - galaxies: active - cosmology: observations

\section{Introduction}

Once considered peculiar and exotic objects, quasars (QSOs) have been recognized in recent years as an important and possibly a necessary phase of galactic evolution, at least for spheroids (Danese et al. 2003). In particular, models of a joint evolution of galaxies and QSOs (e.g. Granato et al. 2001, 2004; Hopkins et al. 2005, 2006) reproduce the properties of present-day elliptical galaxies assuming that their vigorous star formation at high redshift is quenched by the feedback (i.e. galactic winds). This process is triggered by the QSO activity fed by the accretion of matter onto a supermassive black hole (SMBH) at the center of the galaxy itself (see also Monaco \& Fontanot 2005). In this way, the properties of high redshift QSOs and, in particular, their luminosity function (LF) are fundamental in understanding the phenomena driving galaxy formation. It has been hypothesized that the feedback from AGN inverts the dark matter halo $(\mathrm{DMH})$ hierarchical sequence for the collapse of baryonic matter, a phenomenon also known by the term downsizing (e.g. Granato et al. 2004; Croton et al. 2006; Bower et al. 2006; Fontanot et al. 2006; Menci et al. 2006; Scannapieco \& Oh 2004). The depth of the potential wells in different DMH is a key factor in determining the timescale for star formation, accretion, and eventually for the

$\star$ Appendix is only available in electronic form at http: //www . aanda.org effects of feedback, setting the pace for AGN activity throughout cosmic history.

Great progress has recently been achieved thanks to the large amount of data coming from major observational programs such as the Two Degree Field QSO Redshift Survey (2QZ, Croom et al. 2004, www.2dFquasar.org) and the Sloan Digital Sky Survey, with the third edition of the Quasar Catalog (DR3QSO, Schneider et al. 2005). The 2dF-QRS contains more than 23000 QSOs typically at redshift $z \leq 2.1$, while the DR3QSO lists 46420 QSOs up to $z \sim 5.4$. However, at high redshift the SDSS is sensitive only to the most luminous QSOs $\left(M_{145} \lesssim-26.5^{1}\right)$. The faint-end of the high- $z$ QSO LF, which plays a key role in comparing different predictions of the formation and evolution of galaxies, is left almost unconstrained due to the challenging magnitude limit.

The recent Great Observatories Origins Deep Survey (GOODS) provides an important database for studying QSOs with $M_{145} \lesssim-21$, thanks to the depth of its optical observations and to its multi-wavelength nature. GOODS is a deep survey, not a wide one, but it is much larger than most previous deep HST/WFPC2 surveys, covering $320 \mathrm{arcmin}^{2}, 32$ times the combined solid angles of the Hubble Deep Field-North and South, and four times larger than their combined flanking fields. The GOODS-ACS optical data were analyzed in a previous paper

\footnotetext{
1 Absolute $\mathrm{AB}$ magnitude at a wavelength of 145 nanometers.
} 
(Cristiani et al. 2004, hereafter Paper I) in order to select reliable QSO candidates and make a first estimate of their space density in the redshift interval $3.5<z<5.2$. The selection was carried out by defining suitable optical selection criteria based on magnitude limits and color criteria, and then by matching the optical candidates with Chandra X-ray surveys (Alexander et al. 2003; Giacconi et al. 2002).

In this paper we take advantage of the SDSS 3rd Data Release, of a new analysis and of a more complete spectroscopic follow up of the GOODS data in order to determine the QSO space density at $3.5<z<5.2$, down to the faint-end of the LF $\left(M_{145}<-21\right)$. As in Paper I we adopt a definition of QSOs encompassing all objects with strong, high-ionization emission lines and $M_{145} \leq-21$, including conventional, broadlined (type-1) QSOs, and narrow-lined, obscured (type-2) QSOs. Throughout the paper we adopt a flat universe with cosmological parameters $\Omega_{\mathrm{m}}=0.3, \Omega_{\Lambda}=0.7$, and Hubble constant $H_{0}=70 \mathrm{~km} \mathrm{~s}^{-1} \mathrm{Mpc}^{-1}$. Magnitudes are in the AB system.

In Sect. 2 we present the databases, the selection of the QSO candidates, and the spectroscopic follow up; in Sect. 3 we present our algorithm for the determination of the LF; in Sect. 4 we discuss the results in terms of implications for current models of galaxy formation.

\section{The database}

\subsection{Bright QSOs from the SDSS}

The bright quasar sample we use in this work was extracted from the DR3QSO. The main SDSS catalogue covers an area of about $5282 \mathrm{deg}^{2}$ in photometry and $4188 \mathrm{deg}^{2}$ in spectroscopy. Photometric catalogues were compiled with observations in $u, g$, $r, i, z$ bands (Fukugita et al. 1996). Several selection criteria were tailored in order to select QSOs candidates in SDSS at different redshifts (see e.g. Fan et al. 2001). The selection criteria have been changing significantly over time (Schneider et al. 2005). Richards et al. (2002, hereafter R02) present the updated version of these selection criteria applied to select suitable QSO candidates for spectroscopy. We refer to these criteria in the following sections (namely to Eqs. (6) and (7) in R02). In particular we are interested in the selection of high- $z$ objects. Due to the strategy of the SDSS observations, the DR3QSO is a sample with an incomplete follow-up.

Fan et al. (2003) have compiled a complete sample of high- $z$ QSOs out of the SDSS commissioning data; however, we decided to use DR3QSO, in order to have a larger sample of objects selected in a larger area of the sky. The price of this choice consists our being compelled to make a more complex analysis to take the incompleteness of the DR3QSO follow-up into account for our purposes (see Sect. 3). There are 656 QSOs in DR3QSO that have a redshift between $3.5<z<5.2$ and that satisfy R02 criteria. We considered those objects in the SDSS photometric catalogue that satisfy the R02 criteria and we compared them to the corresponding SDSS spectroscopic catalogue. This way, we have estimated that only $\sim 21 \%$ of the candidates satisfying the R02 criteria have a spectrum.

\subsection{Faint $Q S O$ s from the GOODS}

The GOODS covers an area of $320 \mathrm{arcmin}^{2}$, subdivided into two $160 \operatorname{arcmin}^{2}$ sub-fields centered on the Chandra Deep FieldSouth (CDF-S) and Hubble Deep Field-North (HDF-N). The optical data $\left(B_{445}, V_{660}, i_{775}, z_{850}\right.$ bands) were obtained with the Advanced Camera for Surveys (ACS) onboard HST in the framework of the GOODS/ACS survey described in Giavalisco et al. (2004a). The catalogues used in this paper, prepared using the SExtractor package (Bertin \& Arnouts 1996), are based on the version $v 1.0$ of the reduced, calibrated, stacked, and mosaiced images acquired with HST and ACS as part of the GOODS ACS Treasury program ${ }^{2}$. The catalogues are $z$-band-based, that is, source detection was made using the $z$-band images. The magnitude limits in the four bands are 27.50 ( $B_{445}$ band), 27.25 ( $V_{660}$ band), 27.00 ( $i_{775}$ band), and 26.5 ( $z_{850}$ band $)$ at $\mathrm{S} / \mathrm{N} 10$ for point sources.

The HDF-N and CDF-S fields were observed in the X-rays with Chandra for $2 \mathrm{Ms}$ and $1 \mathrm{Ms}$, respectively (Alexander et al. 2003, hereafter A03; Giacconi et al. 2002, hereafter G02), providing the deepest views of the Universe in the $0.5-8.0 \mathrm{keV}$ band. The X-ray completeness limits over $\approx 90 \%$ of the area of the GOODS fields are similar, with flux limits $(S / N=$ 5) of $\approx 1.7 \times 10^{-16} \mathrm{erg} \mathrm{cm}^{-2} \mathrm{~s}^{-1}(0.5-2.0 \mathrm{keV})$ and $\approx 1.2 \times$ $10^{-15} \mathrm{erg} \mathrm{cm}^{-2} \mathrm{~s}^{-1}(2-8 \mathrm{keV})$ in the HDF-N field and $\approx 2.2 \times$ $10^{-16} \mathrm{erg} \mathrm{cm}^{-2} \mathrm{~s}^{-1}(0.5-2.0 \mathrm{keV})$ and $\approx 1.5 \times 10^{-15} \mathrm{erg} \mathrm{cm}^{-2} \mathrm{~s}^{-1}$ $(2-8 \mathrm{keV})$ in the CDF-S field (A03). The sensitivity at the aim point is about 2 and 4 times better for the CDF-S and HDF-N, respectively. As an example, assuming an X-ray spectral slope of $\Gamma=2.0$, a source detected with a flux of $1.0 \times 10^{-16} \mathrm{erg} \mathrm{cm}^{-2} \mathrm{~s}^{-1}$ would have both observed and rest-frame luminosities of $8 \times$ $10^{42} \mathrm{erg} \mathrm{s}^{-1}$ and $3 \times 10^{43} \mathrm{erg} \mathrm{s}^{-1}$ at $z=3$ and $z=5$, respectively (assuming no Galactic absorption). A03 produced point-source catalogues for the HDF-N and CDF-S and G02 for the CDF-S.

\subsubsection{The color selection}

The selection of the QSO candidates was carried out in the magnitude interval $22.25<z_{850}<25.25$. To avoid the lower quality zones at the borders of the GOODS ACS mosaics, we carried out the selection in a slighty reduced area of $157.1 \mathrm{arcmin}^{2}$ in the HDF-N and 156.4 arcmin $^{2}$ in the CDF-S.

Expected QSO colors in the ACS bands were estimated as a function of redshift using a template of Cristiani \& Vio (1990, hereafter CV90) for the QSO spectral energy distribution (SED) convolved with a model of the intergalactic medium (IGM) absorption (see Appendix). The same scheme will be adopted in the following for the estimate of expected colors in the different SDSS passbands, unless otherwise specified.

In Paper I, four optical criteria had been tailored to select QSOs at progressively higher redshift in the interval $3.5 \lessgtr z \lesssim$ 5.2.:

$i-z<0.35$ and $1.1<B-V<3.0$ and $V-i<1.0$

$i-z<0.35$ and $B-V>3.0$

$i-z<0.5$ and $B-V>2.0$ and $V-i>0.8$

$i-z<1.0$ and $V-i>1.9$.

As already shown in Paper I, these color selection criteria are expected to be most complete and reliable at $z>4$ (corresponding to criteria 2-4). These criteria select a broad range of high-z AGN, not limited to broad-lined (type-1) QSOs, and are less stringent than those typically used to identify high- $z$ galaxies (e.g. Giavalisco et al. 2004b). Below $z \simeq 3.5$, the typical QSO colors in the ACS bands move close to the locus of stars and low-redshift galaxies. Beyond $z \simeq 5.2$, the $i-z$ color starts increasing and infrared bands would be needed to identify QSOs efficiently with an " $i$-dropout" technique. The full set of color criteria selects objects in the redshift range $(3.5<z<5.2)$,

\footnotetext{
${ }^{2}$ See, for details http://www.stsci.edu/science/goods/
} 
while the subset (2-4) is able to select objects with $z>4$. To avoid contamination from spurious sources, we limited our selection to $z_{850}$ detections with $S / N>5$.

\subsubsection{Matching the color selection to X-ray catalogs}

The optical candidates selected with the criteria (1-4) were matched with X-ray sources detected by Chandra (A03, G02) within an error radius corresponding to the $3 \sigma$ X-ray positional uncertainty. With this tolerance, the expected number of false matches was five and indeed two misidentifications, i.e. cases in which a brighter optical source lies closer to the X-ray position, were rejected (both in the CDF-S). As already shown in Paper I, given the flux limits of the Chandra surveys, Type-1 QSOs with $M_{145}<-21$ should be detectable up to $z \gtrsim 5.2$, up to an optical-to-X-ray flux ratio $\alpha_{o x} \gtrsim-1.7$. We compare this result with the estimate of $\alpha_{o x}$ statistics given by Steffen et al. (2006, their Table 5). They observe a mean value $\left(\alpha_{o x}=-1.408 \pm 0.165\right)$ at this optical luminosity, lower than our limit. Conversely, any $z>3.5$ source in the GOODS region detected in the X-rays must harbor an $\operatorname{AGN}\left(L_{x}(0.5-2 \mathrm{keV}) \gtrsim 10^{43} \mathrm{erg} \mathrm{s}^{-1}\right)$.

The resulting sample consists of 16 candidates, 10 in CDF-S and 6 in HDF-N (Table 1). With respect to the candidates in Paper I the following differences are found: in the HDF-N (CDF-S) three (one) candidates selected in Paper I disappear from the selection, while three (one) new candidates enter the selection. These differences are due to the improvement both in the photometry and in the astrometry of the v1.0 GOODS/ACS catalogue with respect to v0.5. In particular, a better matching is possible between the optical and the X-ray sources. We discuss the implication of these changes in more detail in the next section.

\subsubsection{Spectroscopic follow-up}

Spectroscopic information for all 16 candidates has been obtained by various sources, as listed in Table 1, particularly in the framework of the GOODS Team Spectroscopic Survey (Vanzella et al. 2005, 2006).

Thirteen ( 5 in the HDF-N and 8 in CDF-S) candidates turn out to be $A G N$, with 11 QSOs at $z>3$ (4 and 7), of which 2 (0 and 2) are identified as Type II. One object (GDS J033229.29275619.5) in particular turns out to be a QSO with a redshift $z=4.759$. This candidate was observed in the framework of the GOODS/FORS2 spectroscopic follow-up (Vanzella et al. 2006) and its reduced spectrum (one-hour exposure with FORS 2 at $E S O-V L T)$ is shown in Fig. 2a. In addition to the already known QSO GDS J123647.97+620941.7 at $z=5.189$ (Barger et al. 2002), GDS J033229.29-275619.5 brings to two the total number of objects in the redshift range $4<z<5$. , where our selection criteria are expected to be most complete and reliable.

If we consider the changes in the selected candidates between Paper I and the present work, we can also draw some interesting conclusions. As shown in the previous section, 3 objects selected in Paper I are not recovered in the present work. Two of these objects turn out to be lower-redshift galaxies. The third object is a confirmed QSO candidate with a redshift $z=2.573$. Our analysis shows that its new colors do not satisfy our criteria anymore. On the other hand, if we consider objects present both in the first and in the present selections, those are confirmed QSOs. Finally, 3 new objects are selected with the updated version of the ACS-catalogue and all are confirmed QSOs. Among them we found an additional candidate (GDS J033240.82-275041.4), which is a low-redshift type-2 AGN.

\subsubsection{Checking the completeness of the $\mathrm{X}$-ray criterion}

It is worth noting that the $z=4.76$ QSO (GDS J033229.29275619.5) is a faint X-ray source that was only selected by matching our optical candidates with the supplementary X-ray catalogue by G02. This brings up the possibility that our selection process might be missing QSOs, in particular at $z \geq 4$, if their X-ray flux is reduced below detectable levels (e.g. by peculiar absorption). To assess this potential problem we replaced the X-ray criterion (Sect. 2.2.2) with a morphological one and applied it to a subset of the color-selected candidates at $z \geq 4$.

The SExtractor software provides a set of parameters quantifying the radius (in pixels) inside which a given percentage of the source flux is concentrated. In the following we fix this percentage at $10 \%$, and we adopt as a diagnostic quantity the related parameter (we refer to it as FLUX_RADIUS). SExtractor also provides a parameter termed CLASS_STAR, that quantifies the stellar appearance of the source. For magnitudes in the range of interest, point-like sources (CLASS_STAR $\simeq 1$ ) and extended sources (CLASS_STAR $\simeq 0$ ) are segregated well, while they tend to mix at fainter magnitudes.

The analysis of the distribution of the FLUX_RADIUS values in our sample of QSO candidates shows that objects with greater FLUX_RADIUS values turn out to be either lower redshift contaminant galaxies or Type-2 QSOs. This parameter is typically lower than 2 for Type-1 QSOs, while Type-2 QSOs show FLUX_RADIUS $<2.5$. Values greater than 2.5 usually correspond to low-redshift galaxies and misidentifications. It is interesting to note that, out of the three candidates that were selected in Paper I and not in the present work (see Sect. 2.2.3) the two having FLUX_RADIUS $>2.5$ turn out to be relatively low-redshift galaxies. This criterion can be strengthened if we also consider the CLASS_STAR parameter. In this way a combined morphological selection has been adopted:

$$
F L U X \_R A D I U S<2.0 \text { AND CLASS_STAR>0.7. }
$$

To distinguish reliable QSO candidates from stars we compute the distance $D_{\text {col }}(z)$ between the point-like candidates and the theoretical locus of quasar colors as a function of redshift at $z \geq$ 4 , in the $(B-V, V-i, i-z)$ space

$D_{\mathrm{col}}(z)=\frac{\sqrt{\sum_{i=1}^{3}\left[\operatorname{col}_{i}^{\mathrm{th}}(z)-\operatorname{col}_{i}^{\mathrm{obs}}\right]^{2}}}{\sqrt{3}}$.

For $B$-band dropouts we consider the distance in the $(V-i, i-z)$ space:

$D_{\mathrm{col}}(z)=\frac{\sqrt{\sum_{i=1}^{2}\left[\operatorname{col}_{i}^{\mathrm{th}}(z)-\operatorname{col}_{i}^{\mathrm{obs}}\right]^{2}}}{\sqrt{2}}$.

We define the following criterion on the minimum distance $D_{\text {col }}^{\min }$ :

$D_{\text {col }}^{\mathrm{min}}<0.25 \mathrm{mag}$.

The application of the criteria (5-6) to the ACS catalogues produces the sample is listed in Table 2, which consists of 2 candidates in the northern field and 6 candidates in the southern one. Among the candidates in Table 2, we find the two $z>4$ QSOs already listed in Table 1. In Fig. 1 we plot the colors of the candidates listed in Table 2. 


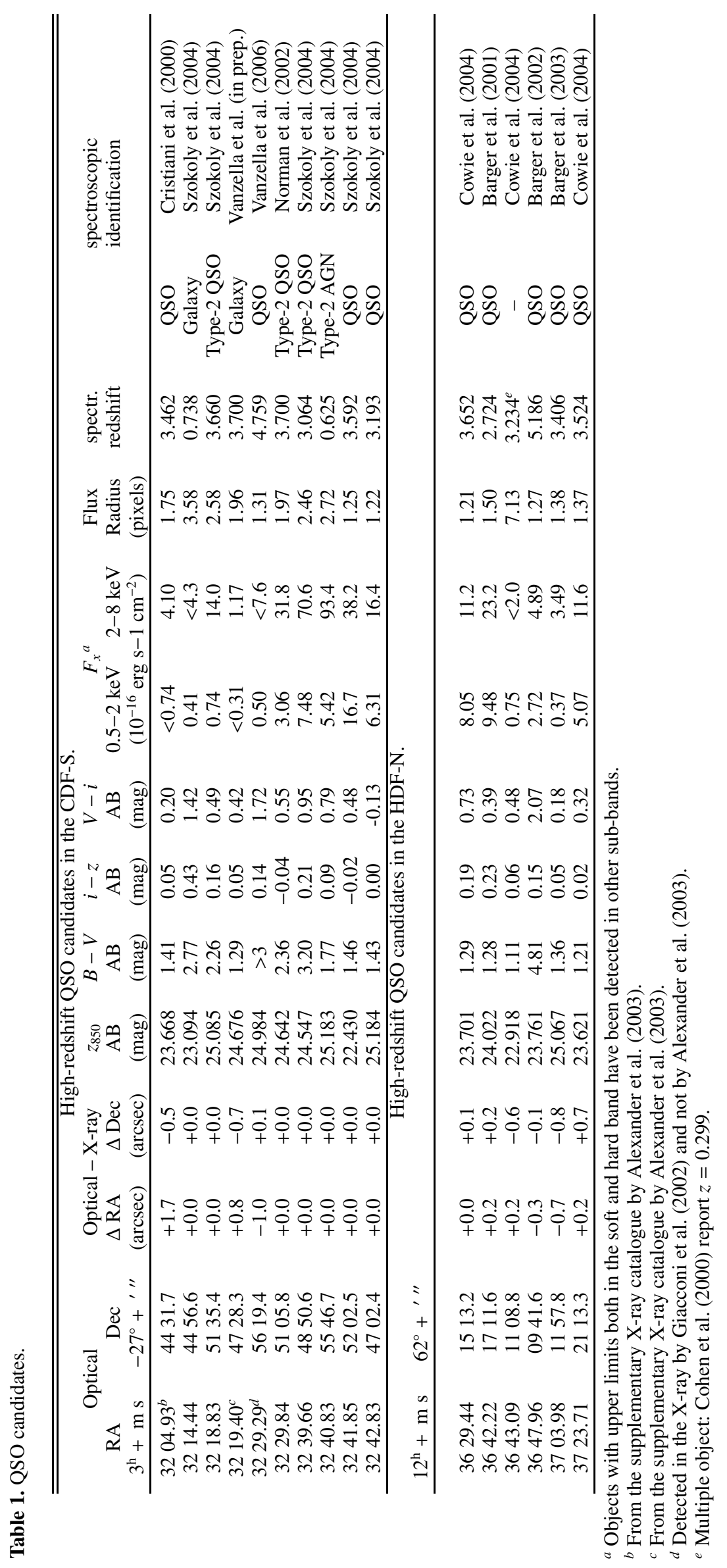




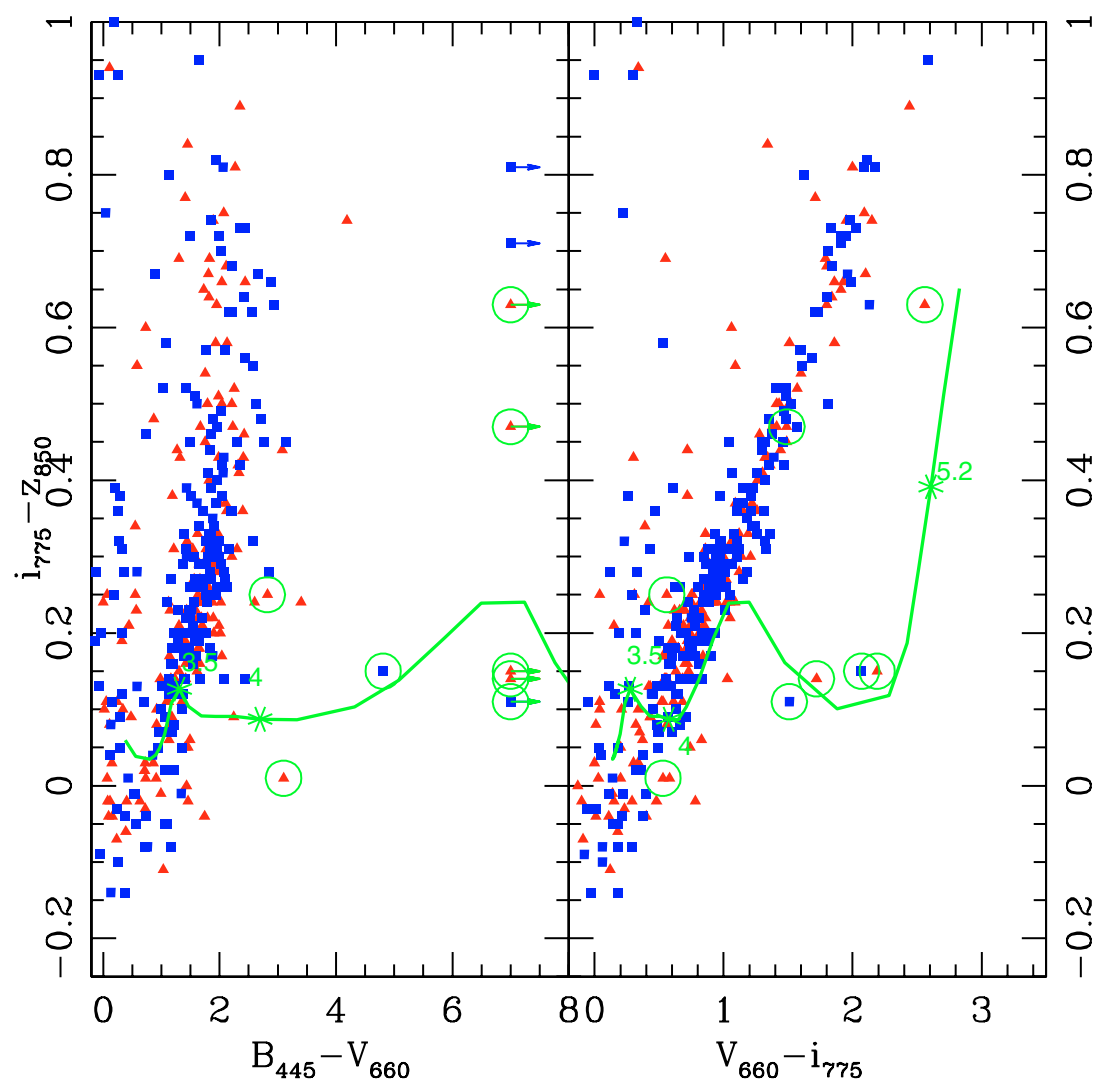

Fig. 1. Left panel: the $B_{445}-V_{660}$ versus $i_{775}-z_{850}$ color in HDF-N and CDF-S. Right panel: the $V_{660}-i_{775}$ versus $i_{775}-z_{850}$ color in HDF-N and CDF-S. The color of point-like candidates are indicated with filled (blue) squares (HDFN) and filled (red) triangles (CDFS). The empty circles mark the positions of our 8 final candidates in the combined fields. The solid line shows the locus of expected QSO colors at $3<z<5.5$; we have marked with asterisks the positions corresponding to redshift $3.5,4$ and 5.2.

A substantial fraction of these objects were observed during GOODS spectroscopic surveys (Vanzella et al. 2006 for the CDF-S, Stern et al. 2006 for HDF-N). The spectra of the CDF-S objects are shown in Fig. 2. Two objects in the CDFS (GDS J033240.38-274431.0 and GDS J033218.91-275302.7) turn out to be high-redshift galaxies, but they lack any evidence of AGN activity. One of them (GDS J033218.91-275302.7) shows an extremely strong $\mathrm{Ly}_{\alpha}$ feature in emission and the [NIV] intercombination line, which, together with the absence of [NV], is suggestive of an H II galaxy (Fosbury et al. 2003) at a redshift $z=5.554$. The spectra of GDS J033201.28-274432.2 and GDS J033216.86-275043.9 show no emission lines. The quality of the spectra is not sufficient to determine a redshift, but enough to exclude their being QSOs in the redshift range $3.5<z<5.2$. The proposed object in HDF-N has been observed in the framework of the GOODS project (D. Stern, private communication). The resulting spectrum is inconclusive, but no sign of AGN activity has been revealed.

On the basis of the morphological selection, no new QSO has been found with respect to the X-ray criterion and no evidence gathered for an incompleteness in the selection of Sect. 2.2.2. The sample listed in Table 1 is therefore considered a reliable estimate of the total QSO space density at $z>3.5$.

\section{Estimating the luminosity function of high-z QSOs}

In this section we describe the procedure we adopted in order to build up the high- $z$ QSO LF from the joint analysis of GOODS and SDSS observations. The two samples are complementary in terms of the surveyed area and the brightness limit and are characterized by different photometric systems, selection criteria, and spectroscopic completeness. In Fig. 3 we show the position of the observed SDSS and GOODS QSOs in the $z-m_{i}$ space (where $m_{i}$ refers to the $i$ magnitude either in the GOODS or SDSS). It is evident from the figure that the two surveys cover two non-overlapping regions of this space.

\subsection{Simulated catalogs}

To combine the GOODS and SDSS information in order to estimate the QSO LF, we adopted the approach of La Franca \& Cristiani (1997, see the appendix for more details on the procedure).

We assume an LF of the form of a double power law:

$\Phi\left(L_{145}, z\right)=\frac{\Phi^{\star}\left(L^{\star}\right)}{\left(L_{145} / L^{\star}\right)^{-\alpha}+\left(L_{145} / L^{\star}\right)^{-\beta}}$

or, expressed in magnitudes:

$\Phi\left(M_{145}, z\right)=\frac{\Phi^{\star}\left(M^{\star}\right)}{10^{0.4(\alpha+1)\left(M_{145}-M^{\star}\right)}+10^{0.4(\beta+1)\left(M_{145}-M^{\star}\right)}}$

We model a pure luminosity evolution (PLE)

$L^{\star}=L_{(z=2.1)}^{\star}[(1+z) / 3.1]^{k_{z}}$

or in magnitude:

$M^{\star}=M_{(z=2.1)}^{\star}-2.5 k_{z} \log [(1+z) / 3.1]$ 


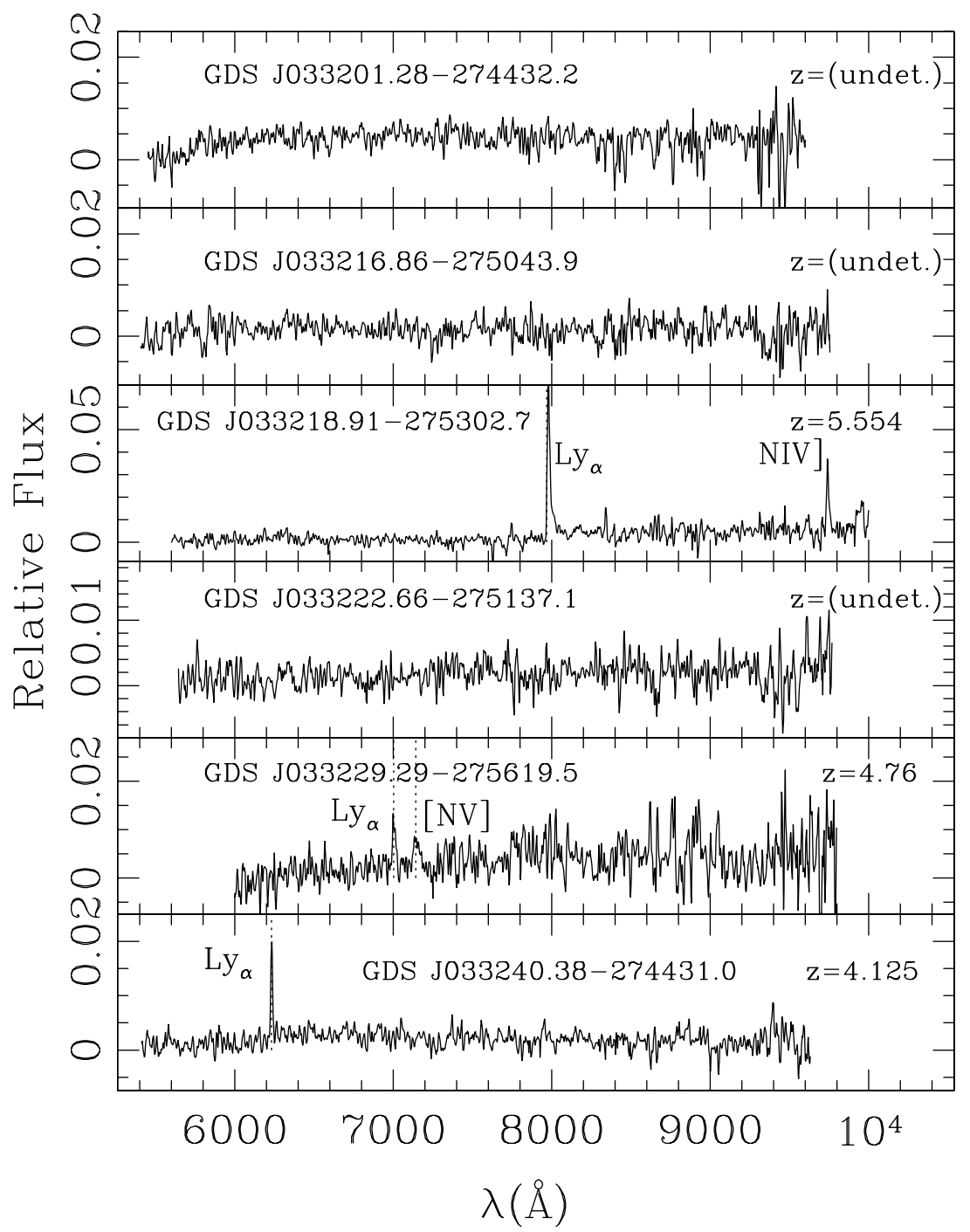

Fig. 2. Spectra of the morphologically+color selected QSO candidates listed in Table 2.

Table 2. Morphologically+color selected QSO candidates.

\begin{tabular}{|c|c|c|c|c|c|c|c|c|c|c|}
\hline \multicolumn{11}{|c|}{ High-redshift QSO candidates in the CDF-S. } \\
\hline \multicolumn{2}{|c|}{ Optical } & \multirow{2}{*}{$\begin{array}{c}z_{850} \\
\mathrm{AB} \\
(\mathrm{mag})\end{array}$} & \multirow{2}{*}{$\begin{array}{c}V-i \\
\mathrm{AB} \\
(\mathrm{mag})\end{array}$} & \multirow{2}{*}{$\begin{array}{c}i-z \\
\mathrm{AB} \\
(\mathrm{mag})\end{array}$} & \multirow{2}{*}{$\begin{array}{c}B-V \\
\mathrm{AB} \\
(\mathrm{mag})\end{array}$} & \multirow{2}{*}{$\begin{array}{l}\text { Class } \\
\text { star } \\
\text { (pixels) }\end{array}$} & \multirow{2}{*}{$\begin{array}{l}\text { Flux } \\
\text { Radius } \\
\text { (pixels) }\end{array}$} & \multirow[b]{2}{*}{ redshift $^{a}$} & \multirow{2}{*}{\multicolumn{2}{|c|}{$\begin{array}{l}\text { spectroscopic } \\
\text { identification }\end{array}$}} \\
\hline $\begin{array}{c}\text { RA } \\
3^{\mathrm{h}}+\mathrm{ms}\end{array}$ & $\begin{array}{c}\text { Dec } \\
-27^{\circ}+{ }^{\prime \prime \prime}\end{array}$ & & & & & & & & & \\
\hline 3201.28 & 4432.2 & 25.206 & 2.19 & 0.15 & $>3$ & 0.75 & 1.97 & 4.85 & & \\
\hline 3216.86 & 5043.9 & 25.190 & 0.56 & 0.25 & 2.82 & 0.92 & 1.39 & 3.81 & & \\
\hline 3218.91 & 5302.7 & 24.561 & 2.56 & 0.63 & $>3$ & 0.85 & 1.53 & 5.554 & EL-Galaxy & Vanzella et al. (2006) \\
\hline 3222.66 & 5137.1 & 25.160 & 1.49 & 0.47 & $>3$ & 0.98 & 1.21 & - & Star & Vanzella et al. (in prep.) \\
\hline 3229.29 & 5619.4 & 24.984 & 1.72 & 0.14 & $>3$ & 0.99 & 1.31 & 4.759 & QSO & Vanzella et al. (2006) \\
\hline 3240.38 & 4431.0 & 25.223 & 0.53 & 0.01 & 3.10 & 0.78 & 1.65 & 4.125 & Galaxy & Vanzella et al. (in prep.) \\
\hline \multirow{2}{*}{\multicolumn{11}{|c|}{ High-redshift QSO candidates in the HDF-N. }} \\
\hline & & & & & & & & & & \\
\hline 3647.96 & 0941.6 & 23.761 & 2.07 & 0.15 & 4.81 & 0.99 & 1.27 & 5.186 & QSO & Barger et al. (2002) \\
\hline 3711.81 & 1133.5 & 25.141 & 1.51 & 0.11 & $>3$ & 0.85 & 1.60 & 4.700 & & \\
\hline
\end{tabular}

${ }^{a}$ Data in italics refer to the photometric estimate of the redshift under the assumption of QSO spectrum.

and, alternatively, a pure density evolution (PDE) with a power law form

$\Phi^{\star}=\Phi_{(z=2)}^{\star}[(1+z) / 3]^{k_{z}}$

or, alternatively, with an exponential form

$\Phi^{\star}=\Phi_{(z=2)}^{\star} \mathrm{e}^{k_{z}[(1+z)-3]}$.
Given a value for the break luminosity $\left(L^{\star}\right.$ or $\left.M^{\star}\right)$, the slopes of the double power law $\alpha$ and $\beta$, the normalization $\Phi^{\star}$, and the redshift evolution parameter $k_{z}$, we are able to calculate the expected number of objects from the LF up to a given magnitude in a given area of the sky. For each object we extract a value of absolute $M_{145}$ and a redshift, according to the 
Table 3. Best fit parameters of the LF of high-redshift QSOs.

\begin{tabular}{|c|c|c|c|c|c|c|c|}
\hline $\begin{array}{c}\text { Model }^{a} \\
\text { Nr. }\end{array}$ & $\begin{array}{c}\text { Free } \\
\text { param. }^{b}\end{array}$ & $\begin{array}{c}\text { Faint } \\
\text { End }\end{array}$ & $\begin{array}{c}\text { Bright } \\
\text { End }\end{array}$ & $M^{\star}$ & $k_{z}$ & $\chi^{2}$ & $P_{\mathrm{KS}}$ \\
\hline \multicolumn{8}{|c|}{ Pure Density Evolution - Power-Law Evolution } \\
\hline 1 & 1 & -1.09 & -3.31 & -25.116 & $-1.71 \pm 0.20$ & 3.2 & $<1 \%$ \\
\hline 2 & 1 & -1.45 & -3.31 & -25.116 & $-1.76 \pm 0.23$ & 3.2 & $<1 \%$ \\
\hline 3 & 2 & -1.09 & -3.31 & $-26.08 \pm 0.11$ & $-4.52 \pm 0.53$ & 1.38 & $<1 \%$ \\
\hline 4 & 2 & -1.45 & -3.31 & $-26.32 \pm 0.11$ & $-5.23 \pm 0.55$ & 0.99 & $<5 \%$ \\
\hline 5 & 2 & -1.58 & -3.31 & $-26.46 \pm 0.22$ & $-5.39 \pm 0.66$ & 0.95 & $5.1 \%$ \\
\hline 6 & 3 & $-1.75 \pm 0.24$ & -3.31 & $-26.48 \pm 0.26$ & $-5.52 \pm 0.94$ & 0.84 & $15 \%$ \\
\hline 7 & 3 & $-1.98 \pm 0.71$ & -2.58 & $-27.06 \pm 2.26$ & $-6.90 \pm 1.36$ & 1.5 & $<1 \%$ \\
\hline \multicolumn{8}{|c|}{ Pure Density Evolution - Exponential Evolution } \\
\hline 8 & 1 & -1.09 & -3.31 & -25.116 & $-0.46 \pm 0.10$ & 2.8 & $<1 \%$ \\
\hline 9 & 1 & -1.45 & -3.31 & -25.116 & $-0.40 \pm 0.15$ & 2.8 & $<1 \%$ \\
\hline 10 & 2 & -1.09 & -3.31 & $-26.11 \pm 0.22$ & $-1.16 \pm 0.18$ & 0.87 & $13 \%$ \\
\hline 11 & 2 & -1.45 & -3.31 & $-26.31 \pm 0.29$ & $-1.27 \pm 0.25$ & 0.63 & $15 \%$ \\
\hline 12 & 2 & -1.58 & -3.31 & $-26.41 \pm 0.39$ & $-1.33 \pm 0.27$ & 0.62 & $16 \%$ \\
\hline $13 \mathrm{a}^{c}$ & 3 & $-1.71 \pm 0.41$ & -3.31 & $-26.43 \pm 0.45$ & $-1.37 \pm 0.46$ & 0.58 & $5.5 \%$ \\
\hline $13 \mathrm{~b}^{d}$ & 3 & $-1.49 \pm 0.24$ & -3.31 & $-26.27 \pm 0.23$ & $-1.26 \pm 0.18$ & 0.65 & $32 \%$ \\
\hline 14 & 3 & $-1.93 \pm 0.31$ & -2.58 & $-26.46 \pm 0.38$ & $-1.42 \pm 0.25$ & 1.3 & $<1 \%$ \\
\hline \multicolumn{8}{|c|}{$\begin{array}{c}\text { Pure Luminosity Evolution } \\
\end{array}$} \\
\hline 15 & 1 & -1.09 & -3.31 & -25.116 & $-0.69 \pm 0.10$ & 2.8 & $<1 \%$ \\
\hline 16 & 1 & -1.45 & -3.31 & -25.116 & $-0.72 \pm 0.11$ & 2.9 & $<1 \%$ \\
\hline 17 & 2 & -1.09 & -3.31 & $-25.78 \pm 0.10$ & $-1.59 \pm 0.21$ & 1.9 & $<1 \%$ \\
\hline 18 & 2 & -1.45 & -3.31 & $-25.82 \pm 0.10$ & $-1.76 \pm 0.23$ & 2.0 & $<1 \%$ \\
\hline 19 & 2 & -1.58 & -3.31 & $-25.91 \pm 0.10$ & $-1.89 \pm 0.20$ & 2.0 & $<1 \%$ \\
\hline 20 & 3 & $-1.31 \pm 0.11$ & -3.31 & $-25.73 \pm 0.25$ & $-1.56 \pm 0.31$ & 1.9 & $<1 \%$ \\
\hline 21 & 3 & $-1.31 \pm 0.35$ & -2.58 & $-26.25 \pm 0.31$ & $-3.47 \pm 0.60$ & 1.0 & $<1 \%$ \\
\hline
\end{tabular}

${ }^{a}$ The normalization of the Luminosity Function is in all cases $\Phi^{\star}=1.67 \times 10^{-6} \mathrm{Mpc}^{-3}$.

${ }^{b}$ Parameters for which no uncertainty is quoted have been fixed in the $\chi^{2}$ minimization.

${ }^{c}$ Parameters estimated through $\chi^{2}$ minimization.

${ }^{d}$ Parameters estimated through $\left(1-P_{\mathrm{KS}}\right)$ minimization.

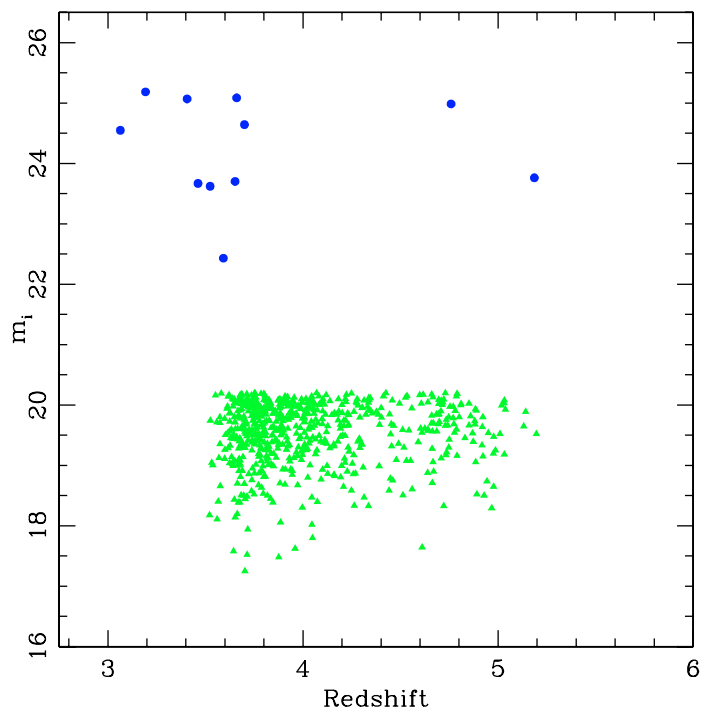

Fig. 3. Distribution of the observed QSOs (SDSS: triangles, GOODS: circles) in the redshift $-m_{i}$ space.

starting parameters of the LF. We then associate a template spectrum to it, randomly chosen from our library (see Appendix A). Using the template k-correction and colors at the corresponding redshift, we simulate the apparent magnitudes in the SDSS and ACS photometric systems. We also add the effect of photometric errors in each band, estimated according to the analysis of the photometric uncertainties as a function of the magnitude in the DR3QSO and GOODS catalogues. In this way we end up with mock SDSS+GOODS catalogues and we then apply to them the selection criteria of Paper I and R02, obtaining selected samples of simulated QSOs.

\subsection{Computing the QSO LF}

The advantage of such an approach is related to being able to generate and analyze a set of simulated SDSS+GOODS samples with the same properties as the real ones in term of selection criteria, completeness, color, and redshift distribution. We can associate simulated catalogues to each starting set of LF parameters. Using multiple realizations for each parameter set, we are also able to limit the statistical error in those simulations, which we can use to estimate the goodness of the agreement of a given LF to the observed sample, by directly comparing observed and simulated objects.

Following the approach described in detail by La Franca \& Cristiani (1997), we divided the $z-m_{i}$ space into bins according to the distribution of the observed objects and we defined a $\chi^{2}$ statistic by comparing the simulated and observed objects in each bin. By using a minimization technique we can then compute the best-fit parameters of the LF.

The functional form of our LF has five free parameters. In previous studies they were estimated in various ranges of redshift. Croom et al. (2004) (hereafter $\mathrm{Cr} 4$ ) have found that the LF is approximated well by a PLE in the redshift range between $0.4<z<2.1$. We use their estimated parameters at $z=2.1$ as a starting point for our PDE and PLE models. We try various possibilities, differing in the number of parameters fitted/fixed and in the type of evolution. As a first attempt we optimized only the evolutionary parameter $k_{z}$ (models listed in Table 3 as 1, 2, $8,9,15,16)$, then we fit two parameters $k_{z}$ and $M_{\star}$ (models 3 , 


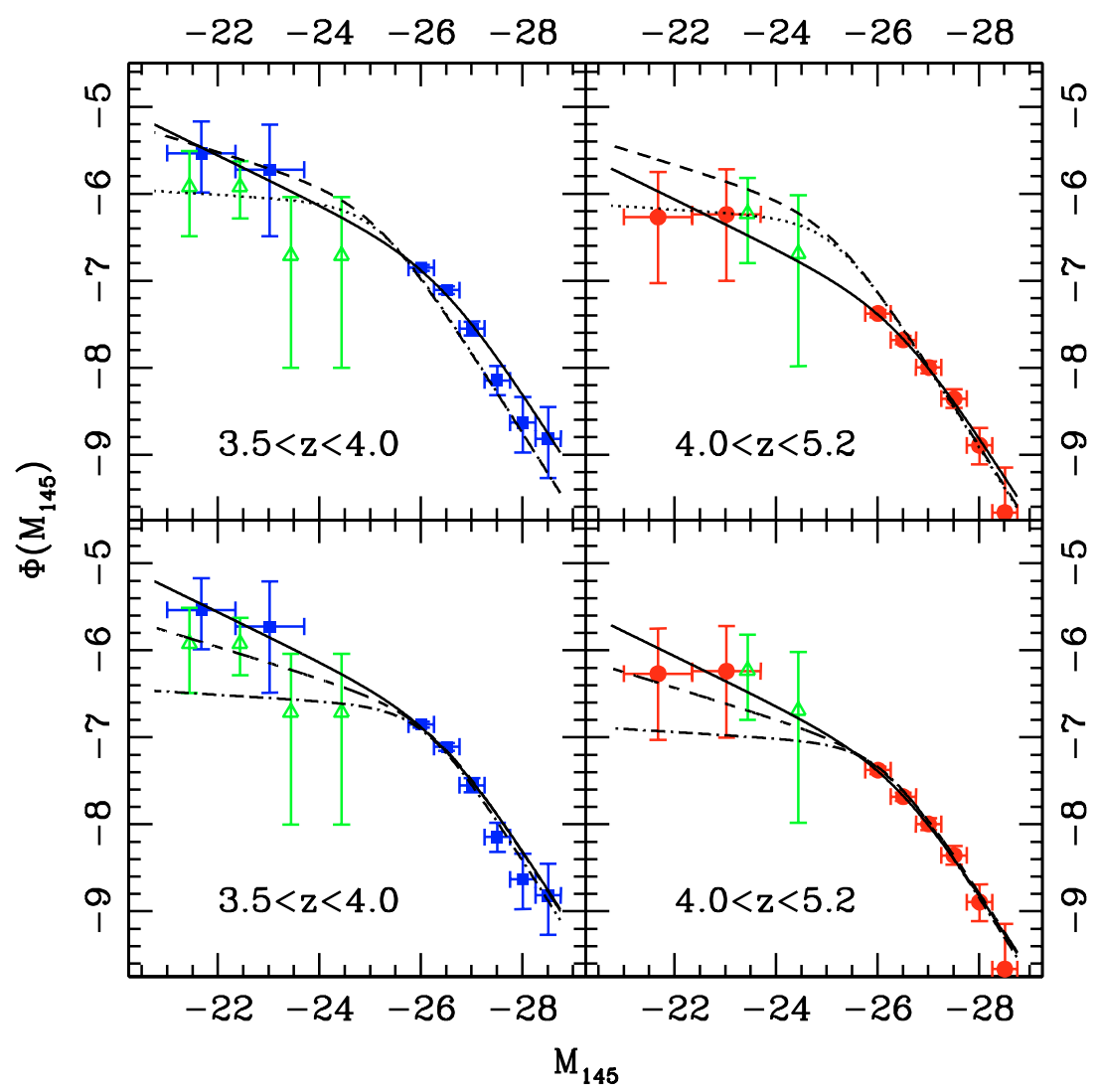

Fig. 4. Analytical fits to the high-z QSO LF: pure density evolution models with exponential evoution. In all panels the solid line shows, as a reference, the model with three free parameters (Nr. 13a in Table 3). Upper panel: models with one free parameter; dotted and dashed lines respectively refer to models with the Croom et al. (2004) and Richards et al. (2005) faint-end (Nrs. 10 and 11). Lower panel: models with two free parameters; dot-dashed and long-short dashed lines respectively refer to models with the Croom et al. (2004) and Richards et al. (2005) faint-end (Nrs. 8 and 9). The position of the filled symbols was obtained by multiplying the values of model Nr. 13a by the ratio between the number of observed sources and the number of simulated sources. La Franca \& Cristiani (1997) demonstrated that this technique is less prone to evolutionary biases with respect to the conventional $1 / V_{\max }$ technique. Empty triangles refer to the results of the COMBO17 survey (Wolf et al. 2003).

$4,5,10,11,12,17,18,19)$, and finally we optimized the three parameters $k_{z}, M_{\star}$ and the faint-end slope $\alpha$ (models $6,13,20$ ).

In a recent paper Richards et al. (2005, hereafter Ri5) compiled a sample of quasars using imaging data from the SDSS and spectra taken by the $2 \mathrm{dF}$ facility at the Anglo-Australian Telescope. Their data are in good agreement with the $2 \mathrm{QZ}$ results of $\mathrm{Cr} 4$ at the bright-end, but they require a steeper faintend slope. We also considered models with the faint-end slope taken from Ri5, $\alpha=1.45$, and the other parameters from $\mathrm{Cr} 4$ (models 2, 4, 9, 11, 16, 18).

Fan et al. (2001) found a shallower slope at high redshift for the bright-end of LF with respect to $\mathrm{Cr} 4$ using SDSS commissioning data. Richards et al. (2006) confirm these findings. At $z<3$ they estimate, in agreement with $\mathrm{Cr} 4$, a bright-end slope $\beta \simeq-3.31$, which progressively flattens to become $\beta \simeq-2.5$ at $z>4$. To take this possibility into account, we also tested models fixing $\beta=-2.58$ and fitting the other parameters (models 7 , $14,21)$.

As a final statistical check of the results, we compared the simulated and observed QSO distributions with a Kolmogorov-Smirnov bidimensional test (see e.g. Fasano \& Franceschini 1987), producing the probability $P_{\mathrm{KS}}{ }^{3}$ listed in the last column of Table 3 .

\footnotetext{
${ }^{3}$ It is worth noting that in general the goodness of fit $\chi^{2}$-wise and $P_{\mathrm{KS}}$-wise is correlated, but the best-fitting parameters are slightly different - though compatible within the uncertainties - when minimizing the $\chi^{2}$ or maximizing the $P_{\mathrm{KS}}$
}

\subsection{Results and discussion}

Table 3 summarizes the results for all the models described in the previous section, indicating for each of them the fixed parameters, the fitted parameters, the corresponding value of the reduced $\chi^{2}$, and the $2-\mathrm{D}$ Kolmogorov-Smirnov probability $P_{\mathrm{KS}}$. The best-fitting models are shown in Fig. 5.

In general it turns out to be impossible to satisfactorily reproduce the observed distribution of objects in the $m-z$ plane with PLE models. On the other hand, PDE models show qualitatively different results according to the number of fixed parameters and the assumed evolution. Models with one free parameter $(1,2,8$, $9,15,16)$ are not able to reproduce the GOODS and SDSS surveys with the assumed $M_{\star}=-25.116$. The agreement becomes satisfactory for PDE models where we fit both $k_{z}$ and $M_{\star}(4,5$, $10,11,12)$. We interpret this result as an indication of $M_{\star}$ evolution from $z \sim 2.1$ to redshifts 3.5-4. We also notice that models with a steeper faint-end slope agree with observations better. This finding is confirmed by models with three free parameters: in particular Nr. 13a, which shows a relatively high probability for a faint-end slope as steep as -1.71 . Models with an exponential evolution of the density tend to agree with the data better than do models with a power-law evolution.

Models with a shallower slope of the bright-end of the LF (as suggested by Fan et al. 2003) are not able to reproduce the observed distributions: a bright-end slope as steep as $\mathrm{Cr} 4$ is required. This result is at variance with Richards et al. (2006), we 


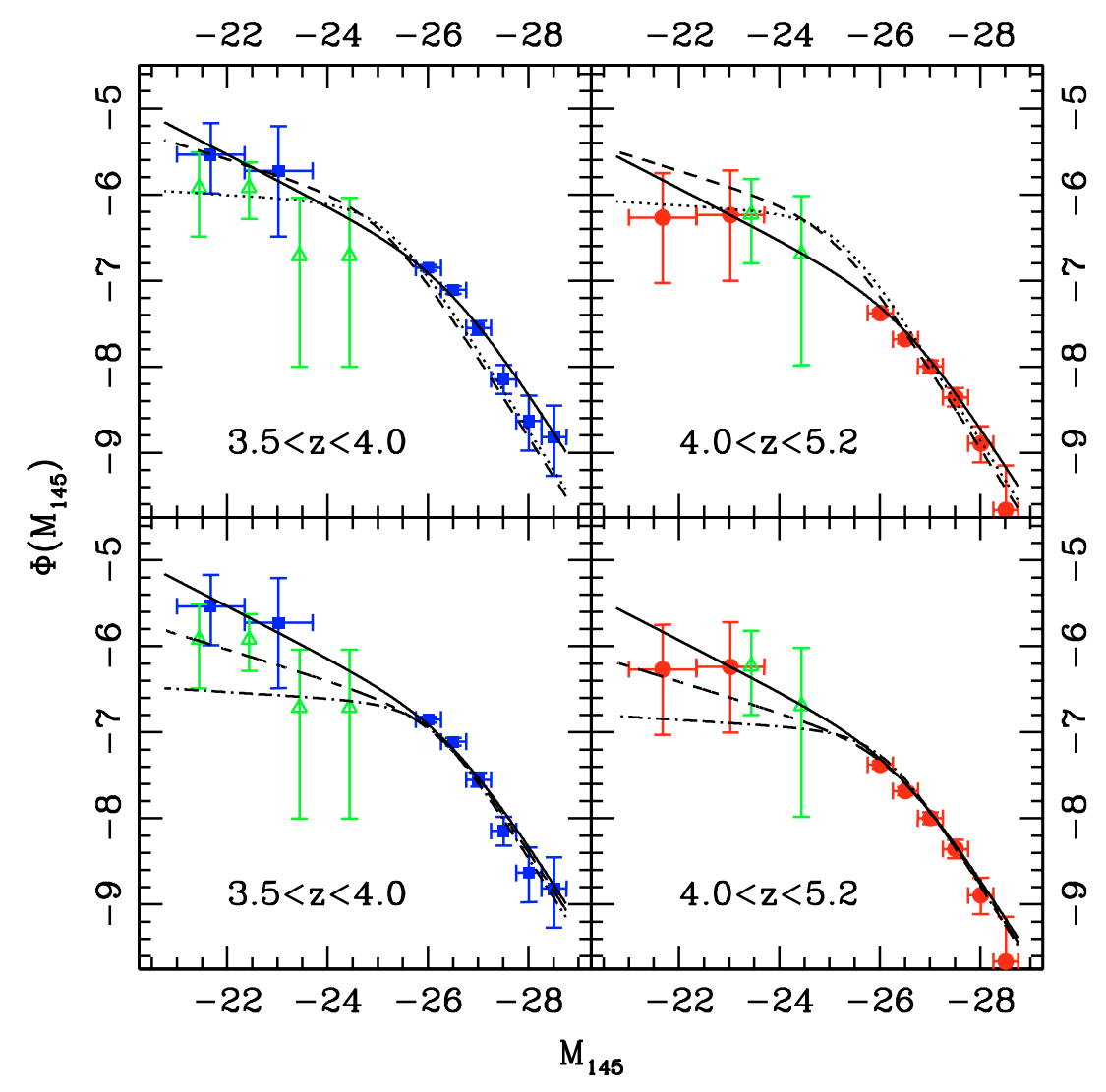

Fig. 5. Analytical fits to the high- $z$ QSO LF: pure density evolution models with power-law evolution. Symbols have the same meaning as in Fig. 4.

ascribe it to the different estimates of the expected QSO colors between them and the present work. As explained in the Appendix, we have defined a QSO template library starting from SDSS spectra in the redshift interval $2.2<z<2.25$. The statistical properties of this library differ from the template spectra used by the SDSS team to calibrate their selection criteria. In particular the mean power-law slope of continuum in our library is redder than the one assumed in Fan (1999). As a consequence (Fig. A.4), we estimate a lower completeness of the SDSS color criteria with respect to Richards et al. (2002), which is, in turn, the main reason for the discrepancy in the estimate of the brightend slope of the LF.

On the other hand, the present results also imply that a slope steeper than $\mathrm{Cr} 4$ and similar to Boyle et al. (2000) is required for reproducing the observed data for the faint end of the LF.

The present observations strengthen the conclusions of Paper I about the space density of high- $z$ quasars and the joint evolution of galaxies and QSOs. Simple recipes, which are based on the assumption that the QSOs shining strictly follows the hierarchical merging structure of DMHs, grossly fail to predict the space density of high- $z$ QSOs. Models trying to account for the complexity of the baryonic matter behavior with respect to DM have to be invoked (see Paper I and references therein for more details about the feedback effects from AGN and stellar formation).

Following the method proposed by Barger et al. (2003), we can use our best estimate for the LF (Nr. 13a) to compute the QSO contribution to the UV background at those redshifts. We calculate the number of ionizing photons per baryon produced in the redshift interval by the observed AGN LF, and we convert the rest frame $1450 \AA$ light to the number density of ionizing photons using the form of the near-ultraviolet spectrum given in Madau (1995). We find a ratio between ionizing photons and baryons on the order of $\sim 0.07$ between $3.5<z<4.0$ and of $\sim 0.02$ at $4.0<z<5.2$. This result is similar to what Barger et al. (2003) found for $5<z<6.5$ QSOs, confirming that the QSO contribution to the UV background is insufficient for ionizing the IGM at these redshifts.

\section{Summary}

In this paper we used the SDSS and GOODS databases to build an LF in the magnitude interval $-21.0<M_{145}<-28.5$ and in the redshift interval $3.5<z<5.2$. In the first part of the paper we completed the selection of the QSOs candidates in the GOODS fields carried out by Paper I. We repeated the original analysis but using an improved version of the optical catalogues, and discuss the results of the spectroscopic follow-up of targets. The main conclusions of Paper I on the relatively low surface density of faint high redshift QSOs are confirmed and strengthened. We also explored the possible presence of "X-ray faint" AGNs, not detected in the A03 and G02 catalogues. This additional search was based on a morphological analysis carried out with the ACS optical images, selecting point-like candidates via a direct comparison with the expected QSO colors. We followed up the seven most promising candidates spectroscopically finding no additional QSO among them and no evidence of any significant population of faint X-ray high- $z$ AGN with magnitude $M_{145}<-21$ in our fields.

We then combined the QSO sample in the GOODS fields with the DR3QSO to study the evolution of the QSO LF. In order to understand the systematics in the different samples, we exploited a method for simulating the combined survey. This 
method is based on a library of QSO template spectra, built up starting from observed SDSS QSO spectra in the redshift interval $2<z<2.25$. Using this library we were able to predict with great reliability the color distributions of observed high- $z$ QSO in the SDSS and GOODS photometric systems. Assuming a parameterization of the $\mathrm{LF}$ and its redshift evolution, we were then able to simulate the expected object distribution in redshift and apparent magnitude. From the statistical comparison between real and simulated samples, we were able to quantitatively evaluate the agreement of the assumed LF with the data.

We tried different LF models and our results point out that PDE models show better agreement with observations than do PLE models, even if a luminosity evolution of the magnitude of the break, $M_{145}^{*}$, from $z=2.1$ to $z=3.5$ is required to obtain a good match. The most interesting result in terms of physical models is related to the faint end slope of the LF. Parameterizations assuming a relatively steep faint-end slope similar to $\mathrm{Ri} 5$ score a higher probability with respect to those with a faint-end slope as flat as in $\mathrm{Cr} 4$. If we also tried fitting also the faint-end slope, we obtain a best-fit value even higher than the Ri5 estimate. A similar conclusion has been reached by Hao et al. (2005). Finally, we do not find any evidence of a flattening at high- $z$ of the bright-end of the LF with respect to the $\mathrm{Cr} 4$ value, at variance with the results of Fan et al. (2003).

We used our best estimate for the LF to compute the QSO contribution to the UV background at redshift $3.5<z<5.2$, and concluded that the QSO contribution is insufficient for ionizing the IGM at these redshifts.

When compared to physically motivated models, the present results indicate a suppression of the formation or feeding of lowmass SMBHs inside DMHs at high redshift. Several processes can lead to this phenomenon: photoionization heating of the gas by UV background (Haiman et al. 1999), feedback from star formation (Granato et al. 2004), or the interaction between the QSO and the host galaxy. In a recent paper, Monaco \& Fontanot (2006) studied this last possibility in detail, providing hints of a new mechanism for triggering galactic winds. In a forthcoming paper (Fontanot et al. 2006), this feedback mechanism will be inserted into a self-consistent model for galaxy and AGN formation (Monaco et al. 2006): the present results for the high- $z$ LF will be used to constrain the model and estimate the relevance of the joint feedback from supernovae and AGN in shaping the redshift evolution of AGNs.

Acknowledgements. We are greatly indebted and grateful to M. Dickinson, D.Stern, P.Tozzi, and the whole GOODS Team for the help in carrying out the observational program, enlightening discussions, and comments. Thanks!

\section{References}

Alexander, D. M., Bauer, F. E., Brandt, W. N., et al. 2003, AJ, 126, 539 (A03) Arnouts, S., Cristiani, S., Moscardini, L., et al. 1999, MNRAS, 310, 540 Barger, A. J., Cowie, L. L., \& Richards, E. A. 2000, AJ, 119, 2092 Barger, A. J., Cowie, L. L., Bautz, M. W., et al. 2001, AJ, 122, 2177 Barger, A. J., Cowie, L. L., Brandt, W. N., et al. 2002, AJ, 124, 1839 Barger, A. N., Garmire, G. P., \& Hornschemeier, A. E. 2003, ApJ, 584, L61 Bertin, E., \& Arnouts, S. 1996, A\&AS, 117, 393

Boyle, B. J., Shanks, T., Croom, S. M., et al. 2000, MNRAS, 317, 1014
Bower, R. G., Benson, A. J., Malbon, R., et al. 2006, MNRAS, 370, 645 Capak, P., Cowie, L. L., Hu, E. M., et al. 2004, AJ, 127, 180

Cohen, J. G., Hogg, D. W., Blandford, R., et al. 2000, ApJ, 538, 29

Cowie, L. L., Barger, A. J., Hu, E. M., Capak, P., \& Songaila, A. 2004, AJ, 127, 3137

Cristiani, S., \& Vio, R. 1990, A\&A, 227, 385 (CV90)

Cristiani, S., Appenzeller, I., Arnouts, S., et al. 2000, A\&A, 359, 489

Cristiani, S., Alexander, D. M., Bauer, F., et al. 2004, ApJ, 600, L119 (Paper I)

Croom, S. M., Smith, R. J., Boyle, B. J., et al. 2004, MNRAS, 349, 1397 (Cr4)

Croton, D. J., Springel, V., White, S. D. M., et al. 2006, MNRAS, 365, 11

Danese, L., Granato, G. L., Silva, L., Magliocchetti, M., \& de Zotti, G. 2003, The Mass of Galaxies at Low and High Redshift, 99

Dawson, S., Stern, D., Bunker, A. J., Spinrad, H., \& Dey, A. 2001, AJ, 122, 598

Dawson, S., Spinrad, H., Stern, D., et al. 2002, ApJ, 570, 92

Dickinson, M. 1998, in The Hubble Deep Field, ed. M. Livio, S. M. Fall, \& P. Madau (Cambridge: Cambridge Univ. Press), 219

Fan, X., Strauss, M. A., Schneider, et al. 2001, AJ, 121, 54

Fan, X., Strauss, M. A., Schneider, D. P., \& Becker, R. H. 2003, AJ, 125, 1649

Fasano, G., \& Franceschini, A. 1987, MNRAS, 225, 155

Fontanot, F., Monaco, P., Cristiani, S., \& Tozzi, P. 2006, MNRAS, accepted [astro-ph/0609823]

Fosbury, R. A. E., Villar-Martín, M., Humphrey, A., et al. 2003, ApJ, 596, 797

Fukugita, M., Ichikawa, T., Gunn, J. E., et al. 1996, AJ, 111, 1748

Giacconi, R., Zirm, A., Wang, J.-X., et al. 2002, ApJS, 139, 369 (G02)

Giavalisco, M., Ferguson, H. C., Koekemoer, A. M., et al. 2004a, ApJ, 600, L93

Giavalisco, M., Dickinson, M., Ferguson, H. C., et al. 2004b, ApJ, 600, L103

Granato, G. L., Silva, L., Monaco, P., et al. 2001, MNRAS, 324, 757

Granato, G. L., De Zotti, G., Silva, L., Bressan, A., \& Danese, L. 2004, ApJ, 600,580

La Franca, F., \& Cristiani, S. 1997, AJ, 113, 1517

Haiman, Z., \& Hui, L. 2001, ApJ, 547, 27

Haiman, Z., Madau, P., \& Loeb, A. 1999, ApJ, 514, 535

Hao, L., et al. 2005, AJ, 129, 1795

Hopkins, P. F., Hernquist, L., Cox, T. J., et al. 2005, ApJ, 630, 705

Hopkins, P. F., Hernquist, L., Cox, T. J., et al. 2006, ApJS, 163, 1

Kennefick, J. D., Djorgovski, S. G., \& de Carvalho 1995, AJ, 110, 2553

Kennefick, J. D., Djorgovski, S. G., \& Meylan, G. 1996, AJ, 111, 1816

Lacey, C., \& Cole, S. 1993, MNRAS, 262, 627

Madau, P. 1995, ApJ, 441, 18

Madau, P., Haardt, F., \& Rees, M. J. 1999, ApJ, 514, 648

Menci, N., Fontana, A., Giallongo, E., Grazian, A., \& Salimbeni, S. 2006, ApJ, 647, 753

Monaco, P., \& Fontanot, F. 2005, MNRAS, 359, 283

Monaco, P., Salucci, P., \& Danese, L. 2000, MNRAS, 311, 279

Monaco, P., Fontanot, F., \& Taffoni, G. 2006, MNRAS, accepted [astro-ph/0610805]

Natali, F., Giallongo, E., Cristiani, S., \& La Franca, F. 1998, AJ, 115, 397

Norman, C., Hasinger, G., Giacconi, R., et al. 2002, ApJ, 571, 218

Press, W. H., \& Schechter, P. 1974, ApJ, 187, 425

Richards, G. T., Fan, X., Newberg, H. J., et al. 2002, AJ, 123, 2945 (R02)

Richards, G. T., Croom, S. M., Anderson, S. F., et al. 2005, MNRAS, 360, 839 (Ri5)

Richards, G. T., Strauss, M. A., Fan, X., et al. 2006, AJ, 131, 2766

Scannapieco, E., \& Oh, S. P. 2004, ApJ, 608, 62

Schmitt, H. R., Kinney, A. L., Calzetti, D., \& Storchi-Bergmann, T. 1997, AJ, 114,592

Schneider, D. P., Hall, P. B., Richards, G. T., et al. 2005, AJ, 130, 367

Songaila, A. 2004, AJ, 127, 2598

Steffen, A. T., Strateva, I., Brandt, W. N., et al. 2006, ApJ, 131, 2826

Stern, D., et al. 2006, in preparation

Szokoly, G. P., Bergeron, J., Hasinger, G., et al. 2004, ApJS, 155, 271

Vanzella, E., Cristiani, S., Fontana, A., et al. 2004, A\&A, 423, 761

Vanzella, E., Cristiani, S., Dickinson, M., et al. 2005, A\&A, 434, 53

Vanzella, E., Cristiani, S., Dickinson, M., et al. 2006, A\&A, 454, 423

Vanzella, E., et al., in preparation

Waddington, I., Windhorst, R. A., Cohen, S. H., et al. 1999, ApJ, 526, L77

Wirth, G. D., Willmer, C. N. A., Amico, P., et al. 2004, AJ, 127, 3121

Wolf, C., Wisotzki, L., Borch, A., et al. 2003, A\&A, 408, 499 
F. Fontanot et al.: The QSO luminosity function at high-z, Online Material $p 1$

\section{Online Material}




\section{Appendix A:}

\section{A.1. QSO template library}

To build up a QSO template library, we decided to use highquality SDSS QSO spectra at lower redshift. We needed a redshift interval for which the SDSS sample had the highest possible level of completeness and the continuum of the QSOs was sampled in the largest possible wavelength interval from the $\mathrm{Ly}_{\alpha}$ emission upward. The interval $2.2<z<2.25$ was chosen on this basis. Among the objects in the DR3QSO with $2.2<z<2.25$, we selected the higher quality spectra. Our final sample consisted of 215 objects. We then estimated the rest-frame spectrum of each object, extending the continuum the blueward of the $\mathrm{Ly}_{\alpha}$ line with the continuum fitting technique derived from Natali et al. (1998). We defined several "continuum windows" along the spectrum and fit the observed fluxes with a power law. We used the resulting parameters to estimate the intrinsic QSO spectrum (before IGM absorption) blueward of the $\mathrm{Ly}_{\alpha}$ line. We used each spectrum in our library to compute the theoretical QSOs colors at increasing redshift in the SDSS and ACS photometric systems. To simulate the QSO spectra at different cosmic epochs, we redshifted our spectra up to the redshift of interest, then used a model for the IGM to simulate the IGM absorption. To test the IGM model predictions at the redshift of interest, we compared them with the observations of Songaila (2004). We applied the IGM model to the CV90 template spectrum and computed the absorption $\tau$ following the same procedure used by Songaila (2004) on real spectra. The result is shown in Fig. A.1. Crosses refer to the observed values of Songaila (2004), while the dashed line represents the prediction of the Madau (1995) model. It is evident from the figure that this model overpredicts the $\mathrm{Ly}_{\alpha}$ transmitted fraction at any redshift higher than 4, and underpredicts the $\mathrm{Ly}_{\beta}$ and $\mathrm{Ly}_{\gamma}$ at redshift higher than 3 . We used the ratio between observations and predictions to compute an empirical correction to the Madau (1995) model at the corresponding wavelengths. The solid line in Fig. A.1 represents the result when we applied our modified IGM absorption model to the CV90 template spectrum.

\section{A.2. QSOs color prediction}

We then computed the expected mean QSO colors using our template library, providing a distribution of colors due to the different continuum slopes and strengths of the emission lines in observed spectra.

In Fig. A.2 we show our results for the SDSS photometric system compared to real colors in DR3QSO. The points refer to the color of observed QSOs at different redshifts (see caption for more details). In the left panel the dashed line refers to the R02 SDSS selection criteria for $z>3.5$ QSOs. Our predictions of QSO colors are represented with solid lines (dotted lines are $5 \%$ and $95 \%$ percentiles of color distribution in the template library. The photometric errors are not accounted for.). The agreement is quite good.

We can also predict the redshift evolution of QSO colors. In Fig. A. 3 we show the $g-r, r-i, i-z$ color (in the SDSS photometric system) as a function of the redshift compared with observed QSO colors. The intrinsic scatter around the mean colors (i.e. not taking photometric errors into account) is shown in Fig. A.2 (the dotted lines refer to $5 \%$ and $95 \%$ percentiles of the color distribution). Similar results hold for the GOODS survey.

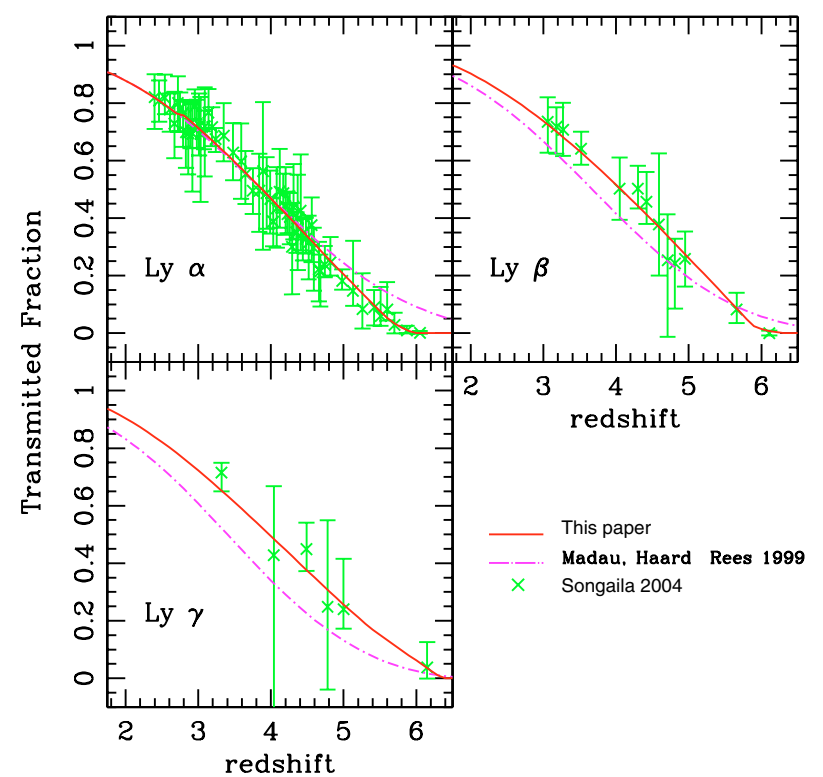

Fig. A.1. Comparison between different estimates of IGM absorption. Crosses (green in the electronic version) refer to Songaila (2004) observations; (magenta) dashed line refers to the prediction of the Madau (1995) model (applied to the QSO template spectrum of CV90), while the (red) solid line shows the applied IGM absorption.

\section{A.3. Completeness against selection criteria}

It is apparent from Figs. A.2 and A.3 that we are able to accurately predict the expected QSO colors as a function of redshift. Our template library can then be used to assess the properties of the two independent samples. In particular we focused our attention on the completeness of the samples selected using the Paper I and R02 criteria. In order to obtain a robust estimate of this quantity we applied the selection criteria to our template library and analyzed the fraction of QSOs recovered at various redshifts. The result is shown in Fig. A.4 (left panel). The SDSS selection criteria shows a lower efficiency in selecting objects at $z \sim 4.4-4.5$. We tried to investigate this issue using the DR3QSO. We considered the redshift distribution of all objects and compared it with the redshift distribution of DR3QSO objects satisfying the R02 selection criteria. The result is shown in Fig. A.4 (right panel). We found a dearth of $z \sim 4.0-4.5$ objects satisfying the selection criteria with respect to the total sample. To estimate its significance, we computed the number of object in DR3QSO between $3.7<z<4.0$ and $4.5<z<5.2$ (677) and the number of object in the same redshift interval satysfing R02 criteria (384). We then rescaled the DR3QSO redshift distribution by the ratio between these numbers, and we ended up predicting 210 QSOs with $4.0<z<4.5$. Only 164 objects satisfying the R02 criteria were actually observed, a discrepancy at $3-\sigma$ level. We also analyzed the same distribution for the Fan et al. (2003) sample, finding a similar result. The result shown in Fig. A.4 (left panel) is different than the analogous plot in Richards et al. (2006), who estimated the completeness of their criteria to be well above $90 \%$ in the whole range of redshift of interest. This discrepancy is due to the different QSO templates adopted. In particular the SDSS selection criteria are tailored on QSO template spectra whose mean continuum slope $f_{v} \propto v^{-\gamma}$ is "bluer" $(\gamma=0.5 \pm 0.3$, Fan 1999) than the mean slope in our template library $(\gamma=0.7 \pm 0.3)$. The inferred completeness has a direct consequence on the shape and evolution of the estimated QSO LF. Assuming the Richards et al. (2006) complete- 
F. Fontanot et al.: The QSO luminosity function at high-z, Online Material p 3
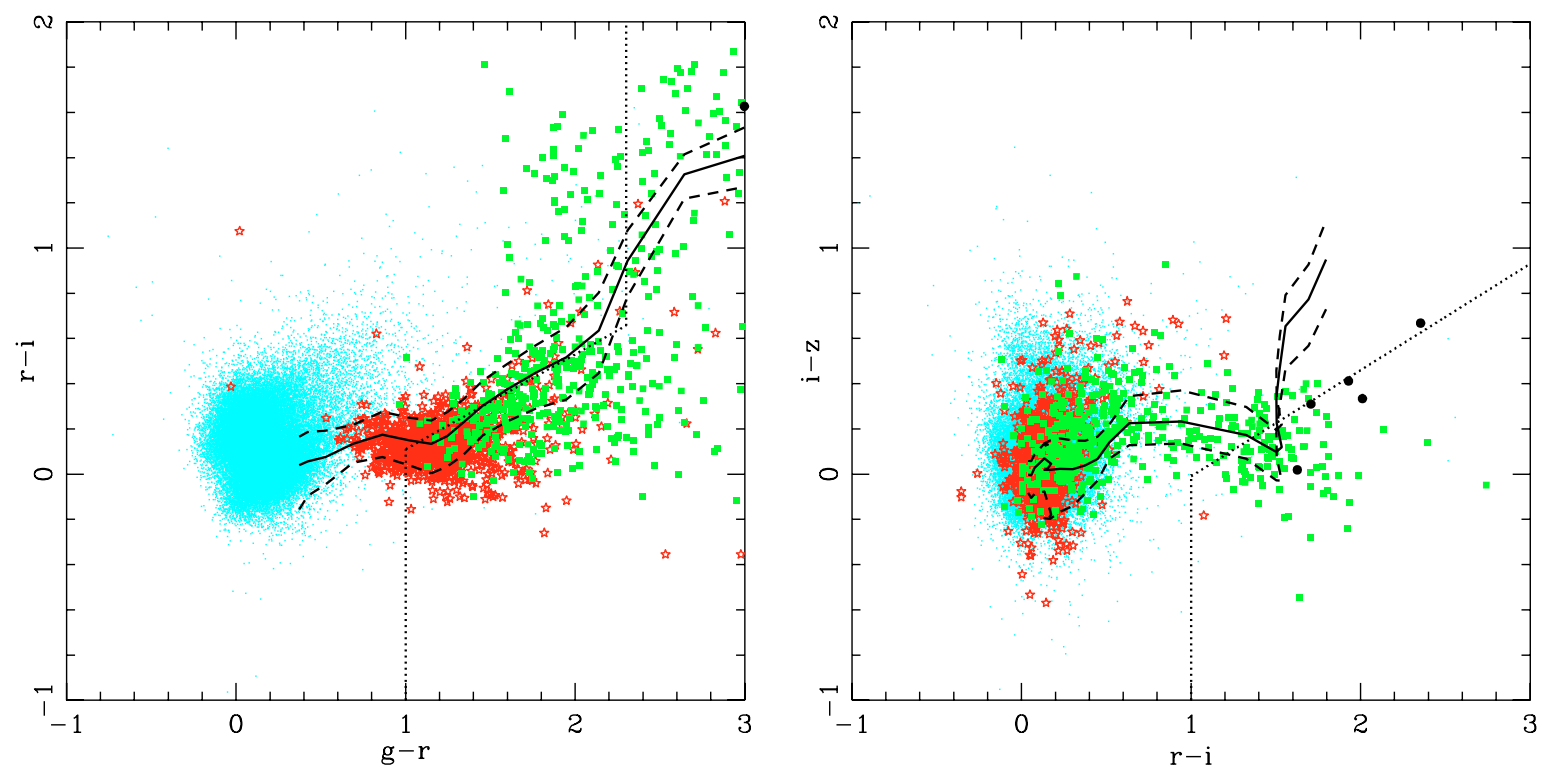

Fig. A.2. Color diagrams for the confirmed QSOs in DR3QSO. Stars (red in the electronic version) refer to objects with $3.5<z<4.0$; (green) squares refer to objects with $4.0<z<5.2$; filled circles refer to objects with $z>5.2$; (cyan) dots refer to objects with $z<3.5$. The solid line shows our prediction of QSO colors in the SDSS photometric system. Dotted lines are the 5\% and 95\% percentiles of color distribution in the template library. The photometric errors are not accounted for. Dashed line shows the selection criteria of Richards et al. (2002) for $z>3.5$ QSOs.

ness, the models with a shallow bright-end (Nr. 7 and Nr. 14) score the highest probability, reproducing the result by Richards et al. (2006). 
F. Fontanot et al.: The QSO luminosity function at high-z, Online Material p 4
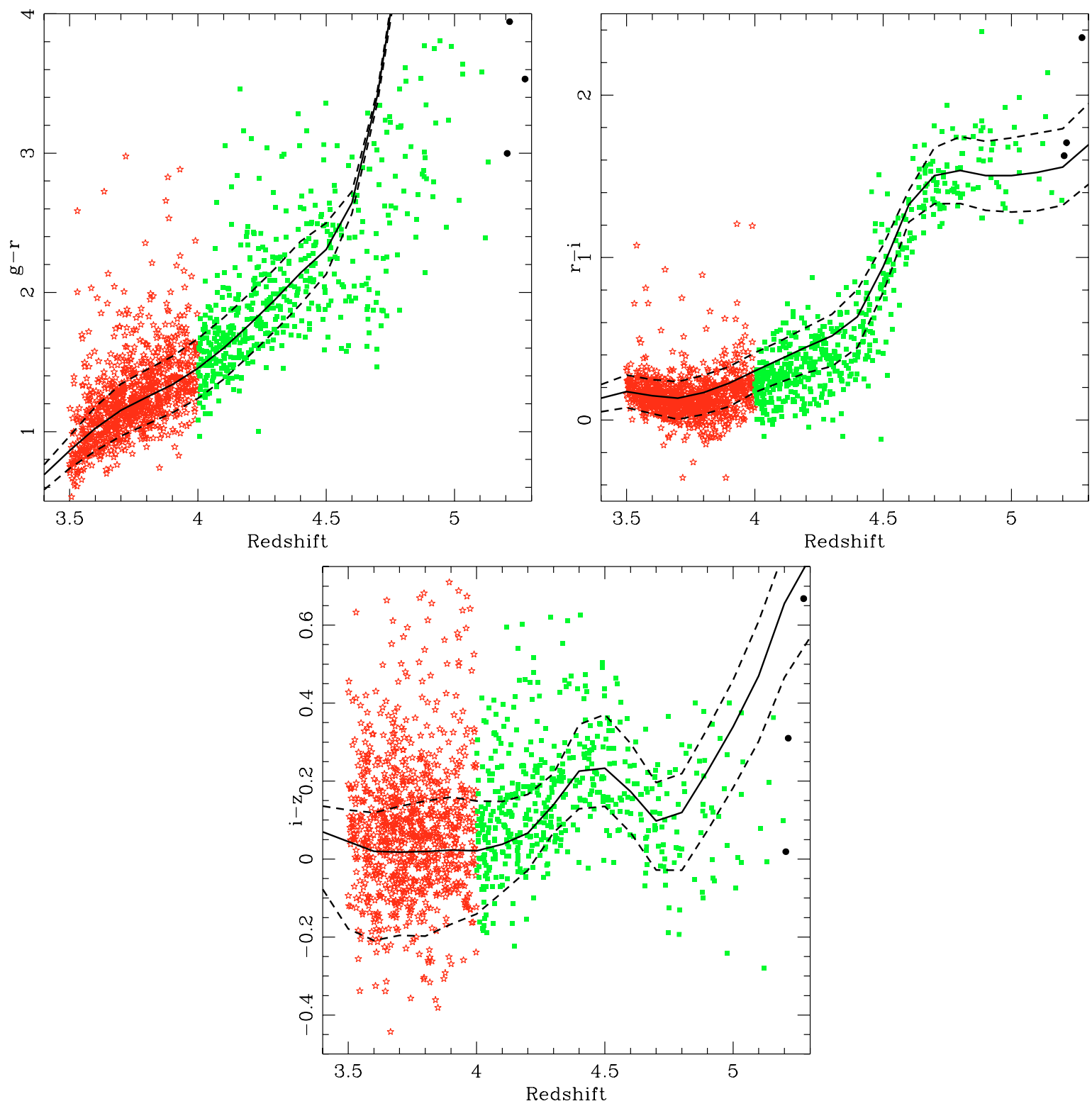

Fig. A.3. Evolution of QSO colors with redshift in the SDSS photometric system. Symbols are the same as for Fig. A.2. 
F. Fontanot et al.: The QSO luminosity function at high-z, Online Material p 5
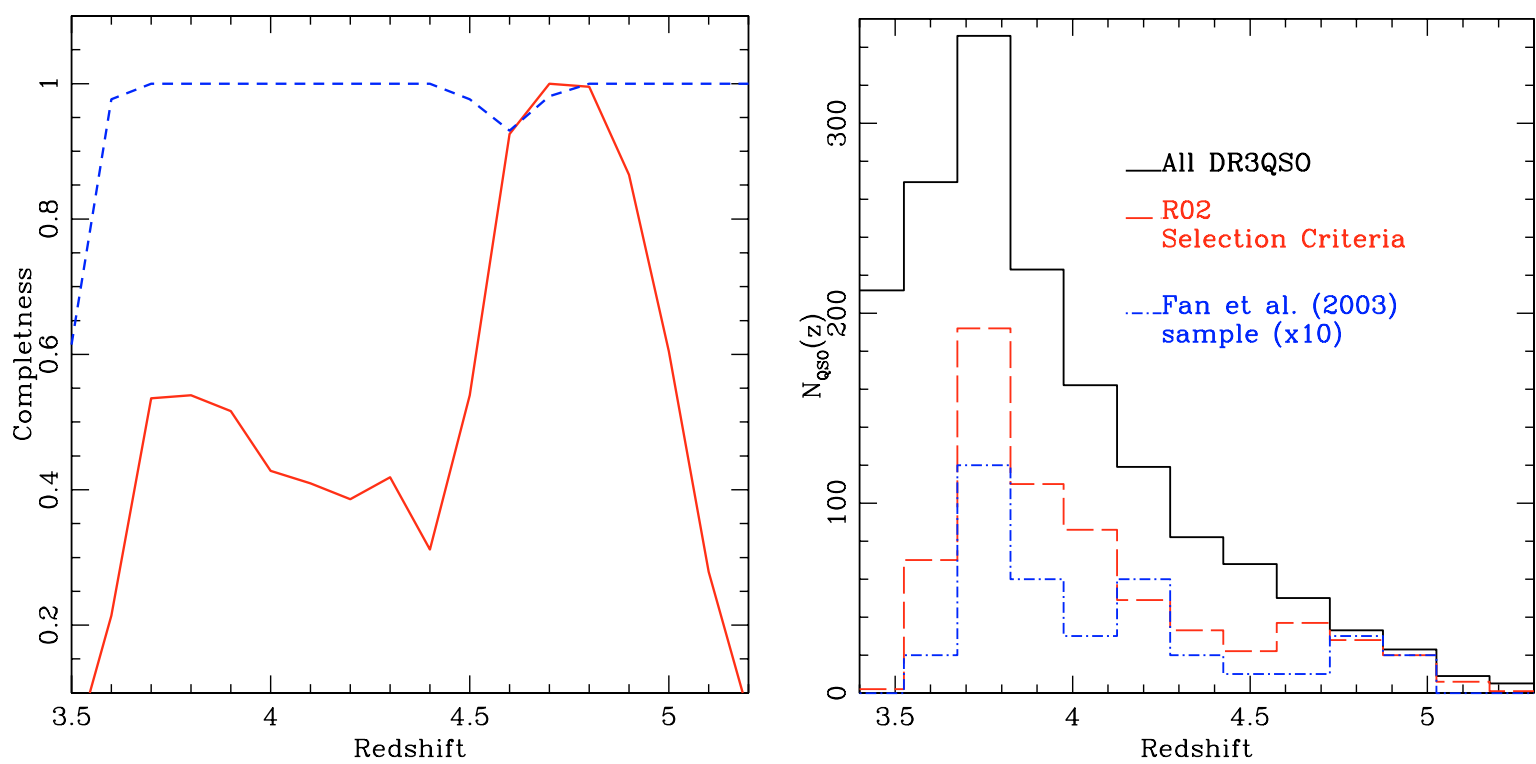

Fig. A.4. Left panel: completeness of selection criteria at various redshifts. The solid line refers to the R02 selection criteria for SDSS sources. The dashed line refers to Paper I selection criteria in the GOODS fields. Right panel: redshift distribution of DR3QSO sources (solid line). Dashed line represents the redshift distribution of DR3QSO sources satisfying the R02 selection criteria. Dotted line represents the redshift distribution of the Fan et al. (2003) sample (multiplied by a factor 10 for graphical reasons) taken out of the SDSS commissioning data. 